\title{
Dexmedetomidine-Ketamine Sedation in a Patient With Dilated Cardiomyopathy
}

\author{
Trent Sims ${ }^{\mathrm{a}, \mathrm{c}}$, Christopher McKee ${ }^{\mathrm{b}}$, Joseph D. Tobias ${ }^{\mathrm{b}}$
}

\begin{abstract}
Sedative and analgesic medications may impact physiologic function resulting in adverse effects especially in patients with co-morbid diseases. We present the successful use of a combination of dexmedetomidine and ketamine to provide sedation and analgesia in a 9-monthold patient with dilated cardiomyopathy undergoing hemodynamic cardiac catheterization with angiography. Co-morbid conditions included depressed myocardial function, hypertension, right renal artery agenesis, and narrowing of the abdominal aorta. A combination of dexmedetomidine and ketamine was used to provide procedural sedation while maintaining spontaneous ventilation. A review of previously published reports of dexmedetomidine and ketamine for procedural sedation is reviewed and the potential efficacy of this combination for procedural sedation in patients with depressed myocardial function is presented.
\end{abstract}

Keywords: Dexmedetomidine; Ketamine; Dexmedetomidine and ketamine sedation; Cardiomyopathy

\section{Introduction}

The American Heart Association defines cardiomyopathies as "a heterogeneous group of diseases of the myocardium associated with mechanical and/or electrical dysfunction that usually exhibit inappropriate ventricular hypertrophy or dilation" [1]. Cardiomyopathy results in ventricular dysfunction which places children at risk for cardiac arrhythmias, heart failure, and multi-organ system dysfunction. Although cardiomyopathy in children is often idiopathic, it may be secondary to congenital heart disease, infection, drugs, genetic disorders or

Manuscript submitted May 25, 2017, accepted June 16, 2017

aDepartment of Pediatrics, Division of Pediatric Critical Care Medicine, Nationwide Children's Hospital and the Ohio State University, Columbus, $\mathrm{OH}$, USA

bDepartment of Anesthesiology \& Pain Medicine, Nationwide Children's Hospital and the Ohio State University, Columbus, OH, USA

${ }^{\mathrm{c} C}$ Corresponding Author: Trent Sims, Nationwide Children's Hospital, 700

Children's Drive, Columbus, OH 43205, USA.

Email: Trent.Sims@Nationwidechildrens.org

doi: https://doi.org/10.14740/jmc2851w other medical conditions. Chronic medical support and frequent diagnostic and invasive procedures are often necessary in the care of children with cardiomyopathy. These procedures often require deep sedation or general anesthesia to ensure patient cooperation, minimize the deleterious physiologic impact of pain, and facilitate successful completion of the procedure. However, patients with significant co-morbid conditions such as depressed myocardial function are at higher risk for complications during the provision of procedural sedation or general anesthesia $[2,3]$.

The provision of adequate sedation and analgesia during such procedures may be problematic given the tenuous nature of myocardial function and the potential impact of sedative and analgesic medications on cardiac output and peripheral vascular resistance. Commonly used agents such as propofol may result in adverse hemodynamic and respiratory effects $[4,5]$. We report our experience with a combination of dexmedetomidine and ketamine for sedation during hemodynamic cardiac catheterization and angiography in a 9-month-old infant with dilated cardiomyopathy and comorbid renal and cardiac involvement. The potential applications of this combination in procedural sedation are discussed and the potential efficacy of this combination in patients with depressed myocardial function is presented.

\section{Case Report}

Approval for the retrospective review and presentation of isolated case reports is not required by the Institutional Review Board at Nationwide Children's Hospital (Columbus, OH, USA). The patient is a former full-term, now 9-month-old, 8.5 -kg male infant who was born via spontaneous vaginal delivery. He had been developing normally until approximately 2 months prior to hospital admission. During this time, he had been having intermittent episodes of emesis with significant weight loss causing him to fall off of the growth curve. The week prior to hospital admission, our patient developed increased emesis with pallor and increased work of breathing, which prompted his presentation to the hospital. Upon evaluation, he was found to be in acute heart failure. His vital signs on presentation included a heart rate (HR) of 155 beats/ min, a respiratory rate (RR) of 52 breaths $/ \mathrm{min}$, a blood pressure (BP) of $91 / 77$, and an oxygen saturation of $98 \%$. Pertinent physical examination findings upon presentation included tachypnea with subcostal retractions, sinus tachycardia with 
S4 gallop, delayed capillary refill at $3 \mathrm{~s}$ and pallor. Chest radiography revealed interstitial pulmonary edema with small pleural effusions and cardiomegaly with left ventricular and right atrial enlargement. The 12-lead electrocardiogram (ECG) showed normal sinus rhythm with right atrial enlargement, left ventricular hypertrophy and T-wave inversion in lateral leads. Transthoracic echocardiogram showed severe dilation of the left ventricle (LV), biventricular dysfunction with an estimated LV ejection fraction of $37 \%$ and an LV fractional shortening of $12.5 \%$, moderate to severe mitral valve regurgitation and trivial tricuspid valve regurgitation with no evidence of elevated pulmonary artery pressures. Patient was admitted to the CTICU for evaluation and management of acute heart failure in the setting of dilated cardiomyopathy. During his hospitalization, following stabilization, he presented for sedation during a hemodynamic cardiac catheterization with angiography.

The patient was kept nil per os for $6 \mathrm{~h}$, during which time maintenance intravenous fluids were provided through a 24-gauge peripheral intravenous cannula. A second 22-gauge peripheral intravenous cannula was inserted uneventfully. Preoperative vital signs included an HR of 109 beats/min, an RR of $40 \mathrm{breaths} / \mathrm{min}$, a BP of 122/86, and an oxygen saturation of $100 \%$. On arrival to the operating room, standard American Society of Anesthesiologists' monitors were placed. Supplemental oxygen $(2 \mathrm{~L} / \mathrm{min})$ was administered and end-tidal carbon dioxide $\left(\mathrm{ETCO}_{2}\right)$ was measured via a nasal cannula. Baseline vital signs in the cardiac catheterization suite revealed an HR of 139 beats/min, a respiratory rate of 19 breaths/min, a $\mathrm{BP}$ of $170 / 81 \mathrm{~mm} \mathrm{Hg}$, an oxygen saturation of $98 \%$, and an $\mathrm{ETCO}_{2}$ of $43 \mathrm{~mm} \mathrm{Hg}$. Bolus doses of dexmedetomidine $(1 \mu \mathrm{g} /$ $\mathrm{kg})$ and ketamine $(2 \mathrm{mg} / \mathrm{kg})$ were administered over $5 \mathrm{~min}$ followed by a dexmedetomidine infusion at $0.2 \mu \mathrm{g} / \mathrm{kg} / \mathrm{h}$ which was titrated up to $0.5 \mu \mathrm{g} / \mathrm{kg} / \mathrm{h}$ for optimal sedation and anxiolysis. Following the initial dose of dexmedetomidine and ketamine, the patient's HR lowered from 139 to 104 beats/ min and his BP lowered from 170/81 to $128 / 53 \mathrm{~mm} \mathrm{Hg}$. An additional $20 \mathrm{mg}$ of ketamine was administered prior to infiltration of the groin and placement of the arterial and venous cannulae. A right heart catheterization, retrograde left heart catheterization, and angiography of the aorta from the aortic valve to just proximal to its bifurcation into the iliac arteries was performed. During the procedure, there were no clinically significant changes in the oxygen saturation, $\mathrm{ETCO}_{2}$ or respiratory rate. The HR varied from 98 to 139 beats $/ \mathrm{min}$ and the BP from $106 / 42$ to $170 / 81 \mathrm{~mm} \mathrm{Hg}$. The lowest BP reading was 106/42 mm Hg. Additional doses of dexmedetomidine $(2 \mu \mathrm{g})$ and ketamine $(10 \mathrm{mg})$ were administered near the end of the case prior to cannula removal. Total sedation time was $117 \mathrm{~min}$. The procedure was completed without difficulty and the patient was uneventfully transported to the post-anesthesia care unit (PACU). In the PACU, the patient required no additional analgesic medications or sedation. He was then returned to the inpatient cardiology unit.

Following the procedure, the patient was hospitalized for an additional 2 weeks for further evaluation and management of his acute heart failure and other medical problems. Further investigation revealed a non-functioning right kidney with elevation of both renin and aldosterone. His dilated cardiomyopathy was thought to be secondary to long-standing hyperten- sion. Follow-up transthoracic echocardiogram was obtained prior to discharge which continued to show severe LV dysfunction with an estimated EF of $32 \%$, severe dilation of the $\mathrm{LV}$, and mild to moderate mitral valve regurgitation. His hypertension was well controlled with oral enalapril. Additional medications for his chronic congestive heart failure included carvedilol and furosemide. As an outpatient, his symptoms of depressed myocardial function have been well controlled with his chronic medication regimen. He continues to gain weight appropriately. The most recent echocardiogram showed resolution of the LV dilation with normal biventricular systolic function and trivial mitral valve regurgitation.

\section{Discussion}

Dilated cardiomyopathy and severe left ventricular dysfunction placed our patient at high risk for adverse effects during procedural sedation as there were both decreased myocardial function and limited cardiac reserve. In a retrospective analysis, Kipps et al evaluated complications after non-cardiac procedures with general anesthesia in 26 children with cardiomyopathy, ranging in age from 5 days to 18.3 years [6]. Approximately half of the patients (46\%) had a complication during the procedure with $83 \%$ of the complications occurring in patients that had severe ventricular dysfunction. Complications included hypotension, arrhythmias, need for mechanical support (extracorporeal membrane oxygenation), and death. Patients with severe ventricular dysfunction, defined as a fractional shortening less than $16 \%$, had a prolonged hospital length of stay $(\mathrm{P}=0.006)$ compared with patients with mild or moderate dysfunction (6 days in severe ventricular dysfunction versus 2 days with mild or moderate dysfunction, $\mathrm{P}=0.006$ ).

Although general anesthesia with endotracheal anesthesia is a viable option for such procedures, endotracheal intubation and positive pressure ventilation can have negative effects on cardiac output [7]. This can include decreased right ventricular (RV) preload, increased RV afterload, decrease LV preload and alterations in ventricular interdependence [8]. Therefore, we chose to use a combination of dexmedetomidine and ketamine to provide procedural sedation with a native airway, thereby avoiding the potential negative impact of positive pressure ventilation.

Dexmedetomidine is an $\alpha_{2}$-adrenergic agonist which initially received FDA approval in the United States in 1999 for the sedation of adults during mechanical ventilation and subsequently in 2009 for monitored anesthesia care (MAC) of adults [6]. While it is FDA-approved only for use in adults, dexmedetomidine has been used safely and successfully in several different clinical scenarios in infants and children including sedation during mechanical ventilation, procedural sedation, supplementation of postoperative analgesia, prevention of emergence delirium, control of post-anesthesia shivering, and the treatment of withdrawal. Although generally effective for sedation during non-invasive procedures, dexmedetomidine as the sole agent has not been uniformly successful for invasive procedures [8-10].

Given the limited analgesic effects of dexmedetomidine 
alone, ketamine was used to provide analgesia especially for the invasive aspects of the procedure such as placement of arterial and venous femoral cannulae [11]. In our patient, a dexmedetomidine loading dose of $1 \mu \mathrm{g} / \mathrm{kg}$ with ketamine $(2 \mathrm{mg} /$ $\mathrm{kg}$ ) administered over 5 min was followed by a dexmedetomidine infusion of $0.2-0.5 \mu \mathrm{g} / \mathrm{kg} / \mathrm{h}$. This provided effective sedation without untoward effects on hemodynamic or respiratory function. Ketamine is often used for the induction of anesthesia in high-risk patients because of its favorable hemodynamic profile which results from the release of endogenous catecholamine stores and the inhibition of neuronal catecholamine reuptake [12]. Rare anecdotal cases of cardiovascular collapse have been reported in patients with severe myocardial dysfunction as ketamine's negative inotropic effects may predominate when endogenous catecholamine stores are depleted by chronic stress or illness [13-15].

Two previous reports have outlined the use of a combination of dexmedetomidine and ketamine during cardiac catheterization $[16,17]$. These and other reports of this combination of agents for procedural sedation have outlined several potential benefits. Most importantly, ketamine will provide analgesia which is limited with dexmedetomidine. With ketamine as part of the regimen for the induction of sedation, the onset times may increase especially in regimens where dexmedetomidine is used as the sole agent with the loading dose administered over $10 \mathrm{~min}$. Dexmedetomidine will blunt the tachycardia, hypertension, salivation, and emergence phenomena from ketamine, while ketamine may prevent the bradycardia and hypotension which has been reported with dexmedetomidine [18].

Although anecdotal reports exist regarding adverse hemodynamic effects of dexmedetomidine in adults with co-morbid cardiac disease, the literature supports the relative safety of dexmedetomidine in pediatric patients with congenital and acquired heart disease including patients following cardiac transplantation [19-23]. Bradycardia is the most concerning of the commonly reported side effects seen with dexmedetomidine. This is particularly concerning in the pediatric population where cardiac output is heart rate dependent. However, the concurrent administration of ketamine attenuates this effect through the centrally mediated sympathetic stimulation and an increase in endogenous catecholamine levels.

Although dexmedetomidine has generally been shown to be devoid of clinically significant effects on respiratory function, occasional episodes of upper respiratory obstruction have been reported [24, 25]. Clinical studies demonstrate fewer effects on central control of ventilation and upper airway patency when compared with propofol [5]. In general, the respiratory effects of dexmedetomidine parallel changes seen during natural sleep. Although there may be changes in minute ventilation, the slope of the $\mathrm{CO}_{2}$ ventilatory response curve is maintained [26].

Although anecdotal, our case illustrates the potential utility of the combination of dexmedetomidine and ketamine for procedural sedation especially in patients with compromised cardiac function. When compared with other agents used for procedural sedation, these two agents should have limited effects on ventilatory function, while their hemodynamic effects should negate each other. Dexmedetomidine can be expected to prevent the tachycardia and hypertension from ketamine, while ketamine prevents the bradycardia and hypotension which has been reported with dexmedetomidine. Ketamine as part of the sedation regimen provides added analgesia which is limited with the use of dexmedetomidine alone.

\section{Conflict of Interest}

The authors of this manuscript have no conflict of interest to disclose.

\section{References}

1. Maron BJ, Towbin JA, Thiene G, Antzelevitch C, Corrado D, Arnett D, Moss AJ, et al. Contemporary definitions and classification of the cardiomyopathies: an American Heart Association Scientific Statement from the Council on Clinical Cardiology, Heart Failure and Transplantation Committee; Quality of Care and Outcomes Research and Functional Genomics and Translational Biology Interdisciplinary Working Groups; and Council on Epidemiology and Prevention. Circulation. 2006;113(14):1807-1816.

2. Malviya S, Voepel-Lewis T, Eldevik OP, Rockwell DT, Wong JH, Tait AR. Sedation and general anaesthesia in children undergoing MRI and CT: adverse events and outcomes. Br J Anaesth. 2000;84(6):743-748.

3. Kipps AK, Ramamoorthy C, Rosenthal DN, Williams GD. Children with cardiomyopathy: complications after noncardiac procedures with general anesthesia. Paediatr Anaesth. 2007;17(8):775-781.

4. Cravero JP, Beach ML, Blike GT, Gallagher SM, Hertzog JH, Pediatric Sedation Research C. The incidence and nature of adverse events during pediatric sedation/ anesthesia with propofol for procedures outside the operating room: a report from the Pediatric Sedation Research Consortium. Anesth Analg. 2009;108(3):795-804.

5. Koroglu A, Teksan H, Sagir O, Yucel A, Toprak HI, Ersoy OM. A comparison of the sedative, hemodynamic, and respiratory effects of dexmedetomidine and propofol in children undergoing magnetic resonance imaging. Anesth Analg. 2006;103(1):63-67, table of contents.

6. Tobias JD. Dexmedetomidine: applications in pediatric critical care and pediatric anesthesiology. Pediatr Crit Care Med. 2007;8(2):115-131

7. Steingrub JS, Tidswell M, Higgins TL. Hemodynamic consequences of heart-lung interactions. J Intensive Care Med. 2003;18(2):92-99.

8. Munro HM, Tirotta CF, Felix DE, Lagueruela RG, Madril DR, Zahn EM, Nykanen DG. Initial experience with dexmedetomidine for diagnostic and interventional cardiac catheterization in children. Paediatr Anaesth. 2007;17(2):109-112.

9. Jalowiecki P, Rudner R, Gonciarz M, Kawecki P, Petelenz M, Dziurdzik P. Sole use of dexmedetomidine has limited utility for conscious sedation during outpatient colonoscopy. Anesthesiology. 2005;103(2):269-273.

10. Alhashemi JA. Dexmedetomidine vs midazolam for monitored anaesthesia care during cataract surgery. Br J 
Anaesth. 2006;96(6):722-726.

11. Tobias JD. Dexmedetomidine and ketamine: an effective alternative for procedural sedation? Pediatr Crit Care Med. 2012;13(4):423-427.

12. Nettles DC, Herrin TJ, Mullen JG. Ketamine induction in poor-risk patients. Anesth Analg. 1973;52(1):59-64.

13. Pagel PS, Kampine JP, Schmeling WT, Warltier DC. Ketamine depresses myocardial contractility as evaluated by the preload recruitable stroke work relationship in chronically instrumented dogs with autonomic nervous system blockade. Anesthesiology. 1992;76(4):564-572.

14. Waxman K, Shoemaker WC, Lippmann M. Cardiovascular effects of anesthetic induction with ketamine. Anesth Analg. 1980;59(5):355-358.

15. Dewhirst E, Frazier WJ, Leder M, Fraser DD, Tobias JD. Cardiac arrest following ketamine administration for rapid sequence intubation. J Intensive Care Med. 2013;28(6):375-379.

16. Tosun Z, Akin A, Guler G, Esmaoglu A, Boyaci A. Dexmedetomidine-ketamine and propofol-ketamine combinations for anesthesia in spontaneously breathing pediatric patients undergoing cardiac catheterization. J Cardiothorac Vasc Anesth. 2006;20(4):515-519.

17. Mester R, Easley RB, Brady KM, Chilson K, Tobias JD. Monitored anesthesia care with a combination of ketamine and dexmedetomidine during cardiac catheterization. Am J Ther. 2008;15(1):24-30.

18. Levanen J, Makela ML, Scheinin H. Dexmedetomidine premedication attenuates ketamine-induced cardiostimulatory effects and postanesthetic delirium. Anesthesiology. 1995;82(5):1117-1125

19. Ingersoll-Weng E, Manecke GR, Jr., Thistlethwaite PA.
Dexmedetomidine and cardiac arrest. Anesthesiology. 2004;100(3):738-739.

20. Sichrovsky TC, Mittal S, Steinberg JS. Dexmedetomidine sedation leading to refractory cardiogenic shock. Anesth Analg. 2008;106(6):1784-1786.

21. Bloor BC, Ward DS, Belleville JP, Maze M. Effects of intravenous dexmedetomidine in humans. II. Hemodynamic changes. Anesthesiology. 1992;77(6):1134-1142.

22. Jooste EH, Muhly WT, Ibinson JW, Suresh T, Damian D, Phadke A, Callahan P, et al. Acute hemodynamic changes after rapid intravenous bolus dosing of dexmedetomidine in pediatric heart transplant patients undergoing routine cardiac catheterization. Anesth Analg. 2010;111(6):14901496.

23. Barton KP, Munoz R, Morell VO, Chrysostomou C. Dexmedetomidine as the primary sedative during invasive procedures in infants and toddlers with congenital heart disease. Pediatr Crit Care Med. 2008;9(6):612-615.

24. Hall JE, Uhrich TD, Barney JA, Arain SR, Ebert TJ. Sedative, amnestic, and analgesic properties of small-dose dexmedetomidine infusions. Anesth Analg. 2000;90(3):699705 .

25. Belleville JP, Ward DS, Bloor BC, Maze M. Effects of intravenous dexmedetomidine in humans. I. Sedation, ventilation, and metabolic rate. Anesthesiology. 1992;77(6):1125-1133.

26. Hsu YW, Cortinez LI, Robertson KM, Keifer JC, SumPing ST, Moretti EW, Young CC, et al. Dexmedetomidine pharmacodynamics: part I: crossover comparison of the respiratory effects of dexmedetomidine and remifentanil in healthy volunteers. Anesthesiology. 2004;101(5):10661076. 